Research Paper

\title{
Identification of miR-4644 as a suitable endogenous normalizer for circulating miRNA quantification in hepatocellular carcinoma
}

\author{
Jun Zhao ${ }^{1,2}$, Xin-Chao Zhu'1,2, Xiao-Song Wu ${ }^{1,2}$, Lin Wang1,2, Can-Can Zhu1, Ke Yang1, Guo-Qing Deng1, An \\ Wang ${ }^{1}$, Yong Liu ${ }^{1}$, Wei-Dong Jia ${ }^{\circledR}$ and Ling Zhu ${ }^{1 凶}$ \\ 1. Center of Engineering Technology Research for Biomedical Optical Instrument, Anhui Institute of Optics and Fine Mechanics, Hefei Institutes of Physical \\ Science, Chinese Academy of Sciences, Hefei 230031, China. \\ 2. Science Island Branch of Graduate School, University of Science and Technology of China, Hefei 230026, China. \\ 3. Department of General Surgery, Anhui Provincial Hospital \& the First Affiliated Hospital of USTC, Division of Life Science and Medicine, University of \\ Science and Technology of China, Hefei 230001, China.
}

$\triangle$ Corresponding author: Ling Zhu, Center of Engineering Technology Research for Biomedical Optical Instrument, Anhui Institute of Optics and Fine Mechanics, Hefei Institutes of Physical Science, Chinese Academy of Sciences, No.350, Shushanhu Road, Hefei 230031, China. Tel/Fax: +86-0551-65392997, E-mail: zhul@aiofm.ac.cn. Wei-Dong Jia, Department of General Surgery, Anhui Provincial Hospital \& the First Affiliated Hospital of USTC, Division of Life Science and Medicine, University of Science and Technology of China, No.17, Lujiang Road, Hefei 230001, China. Tel/Fax: +86-0551-62283740, E-mail: jwd1968@sina.com.

(c) The author(s). This is an open access article distributed under the terms of the Creative Commons Attribution License (https://creativecommons.org/licenses/by/4.0/). See http://ivyspring.com/terms for full terms and conditions.

Received: 2020.05.31; Accepted: 2020.10.04; Published: 2020.10.17

\begin{abstract}
Background: Circulating microRNAs (miRNAs) have proved to be promising biomarkers for early diagnosis and therapeutic monitoring in cancers. Particularly for hepatocellular carcinoma (HCC), detection of circulating miRNA biomarkers as a new diagnostic approach has been written into the latest Guidelines for Diagnosis and Treatment of Primary Liver Cancer in China (2019 edition). However, no general consensus on an ideal endogenous normalizer for circulating miRNAs quantification has been reached, so it will affect the accuracy of quantitative results. In this study, we aim to identify a stable endogenous normalizer for analyzing circulating miRNAs.

Methods: Candidate miRNAs were selected by screening dataset GSE104310, as well as data statistics and analysis. Five commonly reference genes were chosen for further comparison and verification. Then, the expression levels of these genes in serum were analyzed by quantitative reverse transcription PCR (RT-qPCR) among four groups, including patients diagnosed with HCC, chronic hepatitis B (CHB), liver cirrhosis, and healthy subjects. Furthermore, the stability of target genes was evaluated using geNorm, NormFinder, comparative $\Delta \mathrm{Cq}$ programs, and validated by database. We also explored the availability of the miRNA combination, and compared the performance difference between combination and individuals, as well as the selectivity of miRNA references in the combinations.

Results: 11 candidate miRNAs were obtained, and miR-4644 stood out among these miRNAs, and proved to be much more stable than other endogenous miRNAs. Further study showed that miR-4644 exhibited higher stability and expression abundance than other commonly miRNA reference controls. Finally, we discovered the combination of miR-4644 and miR-16 revealed high performance in stability when compared to miRNA individuals. Furthermore, the combination consisted of references with closer nature could give rise to amplification effects in stability.

Conclusions: Our findings demonstrated that miR-4644 is an ideal endogenous normalizer for circulating microRNA quantification in hepatocellular carcinoma. Besides, combining miR-4644 with miR-16 into a whole as a reference control would greatly improve the accuracy of quantification.
\end{abstract}

Key words: circulating microRNA, endogenous control, normalization in RT-qPCR, hepatocellular carcinoma 


\section{Introduction}

Hepatocellular carcinoma (HCC) is one of the most common malignancies that occurs in liver, and accounts for $75 \%$ to $85 \%$ of primary liver cancers [1]. Presently, the morbidity and mortality of HCC are so high that more than half of all new and death cases in the world appeared in China [2,3]. Clinical researches have demonstrated the overall 5-year survival rate was approximately $18 \%$ if diagnosed with terminal liver cancer [4]. However, for early-stage patients, the 5 -year survival rate will exceed $70 \%$ as long as with proper treatment [5]. Thus, early diagnosis and treatment are critical for improving the survival rate of patients. For a long time, serological test, imaging examination, and tissue biopsy were several commonly used detection methods for clinical diagnosis and screening of HCC. But, higher false negative rate [6, 7], lower sensitivity [8] and greater trauma [9] are bottlenecks that restrict the development of these technologies. Therefore, it is particularly important to establish an early-warning system with high-efficiency and precision for the prevention and control of HCC.

MicroRNAs (miRNAs) are a special class of short non-coding RNA that play an important part in developments and physiological activities, and also can act as carcinogenic or tumor suppressor regulators [10,11]. Recently, a growing number of evidence has suggested that circulating miRNAs were highly correlated with a variety of diseases, and changed dynamically with the development of diseases [12-14]. Since 2008, the circulating miRNA was first reported to be a novel biomarker for solid tumors [15], researchers have demonstrated the anomalous variations of expression for circulating miRNAs have occurred in the early stages of a tumor, thus it can be used for risk early warning of tumors in high-risk groups [16, 17]. Currently, the liquid biopsy-based on miRNA molecular markers with high stability, availability, and non-invasion has become an important approach for clinical diagnosis, screening and monitoring $[18,19]$. In other words, it makes it possible to diagnose cancer by a single tube of blood.

Quantitative reverse transcription-polymerase chain reaction (RT-qPCR) is one of the most sensitive methods for miRNA quantification. Generally, with the help of reference genes for data normalization, RT-qPCR could achieve precise quantification of miRNAs. Therefore, the accuracy of expression levels for target miRNAs is heavily relying on the stability of selected reference genes [20]. Presently, endogenous miRNAs, RNU6 (U6), and exogenous miRNA spikeins are several commonly reference controls that used for normalization of miRNA quantification. However, U6 degrades so easily that is fairly unstable in serum, and the research has shown that U6 is not an ideal reference gene for quantifying circulating miRNAs [21]. Certainly for the use of exogenous miRNA spikeins, they may not be able to monitor real level of expression for endogenous miRNAs in real time if the serum samples are taken from patients who have not fasted, so the accuracy of quantitative data for target miRNAs would be seriously affected. By comparison, none of these will happen when employ the endogenous miRNA as reference control. It has been proved to be relatively stable and difficult to degrade in the body fluids, thus, making it the proper reference control for quantification of circulating miRNAs [22, 23]. Recently, there are numerous reports on the use of endogenous miRNA references for analyzing circulating miRNAs, such as miR16 [24], miR-24 [25]. However, there is still no general consensus until now on reference controls for the normalization of miRNAs quantification in cancers $[22,26]$, let alone for analyzing miRNAs in the serum of HCC. Particularly for current diagnosis of HCC, circulating miRNA detection as a new diagnostic approach has been written in the latest Guidelines for Diagnosis and Treatment of Primary Liver Cancer in China (2019 edition). Consequently, it is extremely urgent to find more appropriate endogenous references for accurate quantification of HCC miRNAs.

To our knowledge, there were a few reports on the identification of endogenous miRNAs in HCC, and diverse standpoints in previous researches were also held in scholars. For instance, $\mathrm{Hu}$ et al. identified miR-1288 in plasma, and considered it as a stable endogenous normalizer for quantifying miRNAs in liver patients [27]. However, the so-called stable miR-1288 cannot be detected in the serum array of Lin et al [28], so they had to use synthetic cel-miR-67 for evaluating serum miRNA levels. Given the broad application prospects of miRNA biomarkers used in the field of tumor diagnosis in the future, it is essential to identify a reliable endogenous miRNA for quantitative normalization.

Therefore, in this study, we aim to identify a stable endogenous normalizer for analyzing circulating miRNAs in hepatocellular carcinoma. After integrated analysis on the GEO dataset and TargetScan Human 7.2 database, and validated through clinical samples, candidate miR-4644 was found with high expression stability in serum. Furthermore, several miRNA reference genes (miR16, miR-24, miR-103, miR-191, and U6) were chosen for investigating the performance when compared to miR-4644. Our results suggested that the stability and 
reliability of miR-4644 were better than other references, and the combination of miR-4644 and miR16 was recommended as the optimal normalizer for circulating miRNA quantification.

\section{Materials and Methods}

\section{Patients and ethics statement}

Peripheral blood samples of participants were obtained from four groups, including patients diagnosed with HCC, with chronic hepatitis B, with HBV-induced liver cirrhosis, as well as healthy subjects. All participants were enrolled without any treatments, the patients with any history of cancer were excluded other than HCC, and non-symptoms of liver disease were found in the healthy controls. Furthermore, the recruited participants of these four groups have been confirmed that met the eligibility criteria (Supplementary Table S1). This study had been approved by the Institutional Research Ethics Committee of the Anhui provincial hospital (Ethical No. 2019KY66), and all participants enrolled had signed the written informed consents.

\section{Study design}

Given that the genesis of HCC is resulted from abnormal expression of genes, and expression of genes are regulated by their target miRNAs [29]. Thus, the initial research was carried out and started from key genes of Gene Expression Omnibus (GEO) dataset (https://www.ncbi.nlm.nih.gov/), in order to discover some miRNAs valuable for further in-depth study. Then, the preliminary miRNAs were obtained using TargetScan Human 7.2 database (http:// www.targetscan.org/vert_72/) [30]. Meanwhile, the candidate miRNAs from the subsets of genes were obtained by Venn diagram. Finally, the expression patterns of these candidate miRNAs in the serum samples were verified using RT-qPCR. To confirm the reliability and the stability of potential miRNA references, on one hand, the larger clinical sample size was applied. On the other hand, several commonly reference genes were tested and compared. For verification, the differential expression of these selected miRNAs were analyzed using blood-based biomarkers (BBCancer) database (http://bbcancer. renlab.org/) [31]. Additionally, the comparison between a combination of references and its individuals was made for efficacy evaluation. In brief, the overview of strategy for identifying and evaluating endogenous miRNA normalizers was shown in Figure 1.

\section{Sample preparation and RNA extraction}

For serum preparation, morning peripheral blood was drawn into vacuum tubes, and kept at room temperature at least $60 \mathrm{~min}$, followed been centrifuged at $1000 \times \mathrm{g}$ for $10 \mathrm{~min}$ at $4{ }^{\circ} \mathrm{C}$. Then, the supernatants were transferred to $2.0 \mathrm{~mL}$ sterile tubes, and stored at $-80{ }^{\circ} \mathrm{C}$ for subsequent assays. The procedures of this pretreatment were carried out in accordance with workflow [32]. Total RNA was separated and purified using TRIzol LS reagent and Phasemaker tubes complete system (Invitrogen, California, USA) according to the operation manual. In particular, RNA spike-in kit (QIAGEN, Hilden, Germany) was used for controlling the quality of RNA isolation. The concentrations and quality of pure RNA were measured by NanoDrop 2000 (Thermo Scientific, Wilmington, USA) and the RNA obtained with a value of $260 / 280 \geq 1.80$ would be used in subsequent experiments.

\section{RT-qPCR}

For cDNA synthesis, a total of 50 ng total RNA per sample was transcribed to cDNA using Mir-X miRNA First-Strand Synthesis Kit (Clontech, California, USA), with a $10 \mu \mathrm{L}$ reaction system for one hour at $37{ }^{\circ} \mathrm{C}$, then, the reaction was terminated at $95^{\circ} \mathrm{C}$ for $1 \mathrm{~min}$. The sequences of primers used throughout this study were shown in Table S2. Ultimately, the expression profile of cDNA was analyzed by qPCR using Mir-X miRNA RT-qPCR TB Green kit (Clontech, California, USA). The brief PCR protocol was as follows: denaturation at $95^{\circ} \mathrm{C}$ for $10 \mathrm{~s}$, then, conducted 45 cycles, and each cycle was about 5 $\mathrm{s}$ at $95^{\circ} \mathrm{C}$, as well as $20 \mathrm{~s}$ at $60^{\circ} \mathrm{C}$. Finally, a melting curve was conducted for analyzing the specificity of the amplified products. All the reactions involved were run on LightCycler 96 (Roche, Basel, Switzerland), and each data was repeated three times.

\section{Analysis of gene stability}

Stability of candidate miRNAs expression among different samples was analyzed using three tools, such as geNorm [33], NormFinder [34], and comparative $\Delta \mathrm{Cq}$ method [35], they all using the respective algorithms to verify the stability of target genes. GeNorm was the tool to determine the most stable references by calculating an average expression stability coefficient ( $M$ value), the genes with high $M$ value were gradually eliminated until the pair with the lowest $M$ value was left, and the $M$ value was recalculated for the remaining candidate genes after each high $\mathrm{M}$ value gene was excluded. Furthermore, geNorm can also determine the exact amount of reference controls utilizing analyzing pairwise variable coefficient ( $V$ value), that is when $V_{n} / V_{n+1}<$ 0.15 , the reference genes of $\mathrm{n}$ can satisfy the requirements of the experiment. NormFinder was a procedure based on analysis of variance for sorting 
the stability of genes, and it determined the more stable genes according to the lower $M$ value, the principle was similar to the geNorm stated above. The comparative $\Delta \mathrm{Cq}$ method was an approach based on the total mean of $\mathrm{Cq}$ values in each given sample to normalize every miRNA data, then, the stability per miRNA was evaluated by the mean of standard deviations values of all samples. In particular, the stability varies inversely with the $\mathrm{SD}$ value. So the smaller the value, the better the stability; and this principle could be applied to other algorithms stated above, without exception.

\section{Statistical analysis and normalization}

Student's $t$-test was used to evaluate the difference of miRNA expression between two groups
(HCC vs healthy), and one-way ANOVA and nonparametric tests served for comparisons among four groups (HCC vs $\mathrm{CHB}$ vs liver cirrhosis vs healthy). Particularly, $p$ value $<0.05$ was considered statistically significant. To compare the expression difference of has-miR-4644, as well as other commonly reference genes, relative expression levels were measured by $2^{-\Delta \mathrm{Cq}}$ between HCC and health groups, and the data were normalized to spiked $c$. elegans miR-67 (cel-miR-67). All statistical analyses and graphics were performed by GraphPad Prism version 7.04 (San Diego, CA, USA). Standard deviation (SD), interquartile range (IQR), and CV value were calculated for $\mathrm{Cq}$ values of those genes to evaluate the degree of dispersion between groups.

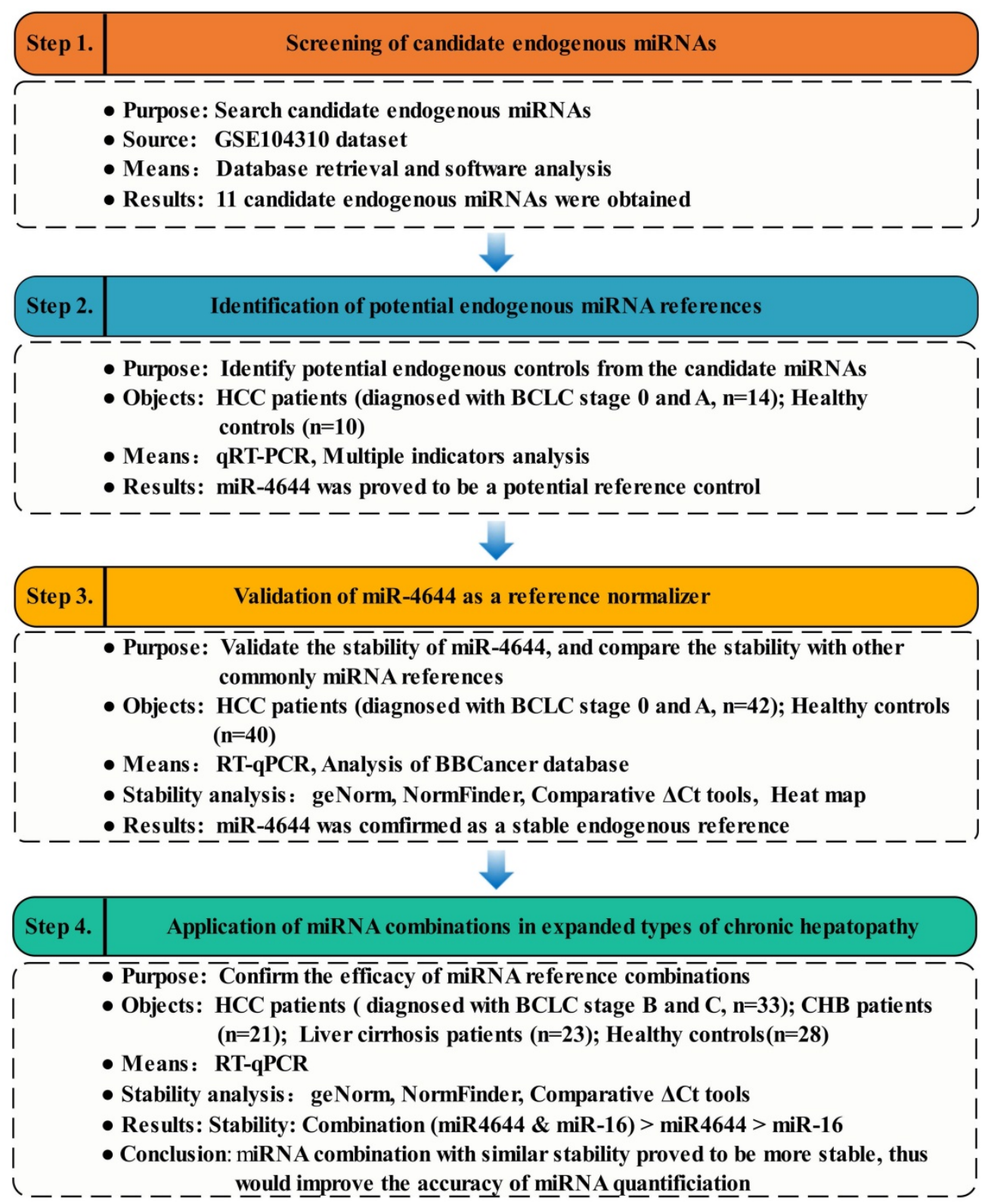

Figure 1. Workflow diagram illustrating strategy for identifying and evaluating endogenous miRNA normalizers. 
Table 1. The clinical characteristics of participants recruited in this study

\begin{tabular}{|c|c|c|c|}
\hline Clinical variable & $\begin{array}{l}\text { Identification stage } \\
\text { (step2, n=24) }\end{array}$ & $\begin{array}{l}\text { Validation stage } \\
\text { (step3, n=82) }\end{array}$ & $\begin{array}{l}\text { Application stage } \\
\text { (step3, n=105) }\end{array}$ \\
\hline HCC group, $n$ & 14 & 42 & 33 \\
\hline \multicolumn{4}{|l|}{ Age (years), $\mathbf{n}$} \\
\hline$<60$ & $11(78.6 \%)$ & $34(81.0 \%)$ & $15(45.5 \%)$ \\
\hline$\geq 60$ & $3(21.4 \%)$ & $8(19.0 \%)$ & $18(54.5 \%)$ \\
\hline \multicolumn{4}{|l|}{ Gender, $\mathbf{n}$} \\
\hline Female & $4(28.6 \%)$ & $10(23.8 \%)$ & $8(24.2 \%)$ \\
\hline Male & $10(71.4 \%)$ & $32(76.2 \%)$ & $25(75.8 \%)$ \\
\hline \multicolumn{4}{|l|}{ ALT, $n$} \\
\hline Low $(\leq 40 \mathrm{U} / \mathrm{L})$ & $9(64.3 \%)$ & $27(64.3 \%)$ & $22(66.7 \%)$ \\
\hline High (> $40 \mathrm{U} / \mathrm{L})$ & $5(35.7 \%)$ & $15(35.7 \%)$ & $11(33.3 \%)$ \\
\hline \multicolumn{4}{|l|}{$\mathrm{AFP}, \mathrm{n}$} \\
\hline Low $(\leq 400 \mathrm{ng} / \mathrm{mL})$ & $10(71.4 \%)$ & $29(69.0 \%)$ & $7(21.2 \%)$ \\
\hline High (> $400 \mathrm{ng} / \mathrm{mL})$ & $4(28.6 \%)$ & $13(31.0 \%)$ & $26(78.8 \%)$ \\
\hline \multicolumn{4}{|l|}{ Tumor size, $\mathrm{n}$} \\
\hline Small $(\leq 5 \mathrm{~cm})$ & $13(92.9 \%)$ & $38(90.5 \%)$ & 0 \\
\hline Large $(>5 \mathrm{~cm})$ & 0 & 0 & $33(100 \%)$ \\
\hline NA & $1(7.1 \%)$ & $4(9.5 \%)$ & 0 \\
\hline CHB group, $n$ & & & 21 \\
\hline \multicolumn{4}{|l|}{ Age (years), $\mathrm{n}$} \\
\hline$<60$ & & & $19(90.5 \%)$ \\
\hline \multirow{2}{*}{\multicolumn{4}{|c|}{$\begin{array}{l}\geq 60 \\
\text { Gender, } \mathbf{n}\end{array}$}} \\
\hline & & & \\
\hline \multicolumn{3}{|l|}{ Female } & $9(42.9 \%)$ \\
\hline \multicolumn{3}{|l|}{ Male } & $12(57.1 \%)$ \\
\hline \multicolumn{4}{|l|}{ ALT, $\mathbf{n}$} \\
\hline \multicolumn{3}{|l|}{ Low $(\leq 40 \mathrm{U} / \mathrm{L})$} & $18(85.7 \%)$ \\
\hline \multicolumn{3}{|l|}{$\operatorname{High}(>40 \mathrm{U} / \mathrm{L})$} & $3(14.3 \%)$ \\
\hline \multicolumn{4}{|l|}{$\mathrm{AFP}, \mathrm{n}$} \\
\hline \multicolumn{3}{|l|}{ Low $(\leq 400 \mathrm{ng} / \mathrm{mL})$} & $21(100 \%)$ \\
\hline \multicolumn{3}{|l|}{ High $(>400 \mathrm{ng} / \mathrm{mL})$} & 0 \\
\hline \multicolumn{3}{|l|}{ Liver cirrhosis group, $n$} & 23 \\
\hline \multicolumn{4}{|l|}{ Age (years), $\mathrm{n}$} \\
\hline \multicolumn{3}{|l|}{$<60$} & $18(78.3 \%)$ \\
\hline \multicolumn{3}{|l|}{$\geq 60$} & $5(21.7 \%)$ \\
\hline Gender, $n$ & & & \\
\hline Female & & & $7(30.4 \%)$ \\
\hline Male & & & $16(69.6 \%)$ \\
\hline ALT, $n$ & & & \\
\hline Low $(\leq 40 \mathrm{U} / \mathrm{L})$ & & & $20(87.0 \%)$ \\
\hline High (> $40 \mathrm{U} / \mathrm{L})$ & & & $3(13 \%)$ \\
\hline $\mathrm{AFP}, \mathrm{n}$ & & & \\
\hline Low $(\leq 400 \mathrm{ng} / \mathrm{mL})$ & & & $23(100 \%)$ \\
\hline High (> $400 \mathrm{ng} / \mathrm{mL}$ ) & & & 0 \\
\hline Healthy group, $n$ & 10 & 40 & 28 \\
\hline Age (years), $\mathrm{n}$ & & & \\
\hline$<60$ & $9(90 \%)$ & $35(87.5 \%)$ & $26(92.9 \%)$ \\
\hline$\geq 60$ & $1(10 \%)$ & $5(12.5 \%)$ & $2(7.1 \%)$ \\
\hline Gender, $n$ & & & \\
\hline Female & $4(40 \%)$ & $12(30 \%)$ & $9(32.1 \%)$ \\
\hline Male & $6(60 \%)$ & $28(70 \%)$ & $19(67.9 \%)$ \\
\hline ALT, $n$ & & & \\
\hline Low $(\leq 40 \mathrm{U} / \mathrm{L})$ & $10(100 \%)$ & $40(100 \%)$ & $28(100 \%)$ \\
\hline $\operatorname{High}(>40 \mathrm{U} / \mathrm{L})$ & 0 & 0 & 0 \\
\hline $\mathrm{AFP}, \mathrm{n}$ & & & \\
\hline Low $(\leq 400 \mathrm{ng} / \mathrm{mL})$ & $10(100 \%)$ & $40(100 \%)$ & $28(100 \%)$ \\
\hline High $(>400 \mathrm{ng} / \mathrm{mL})$ & 0 & 0 & 0 \\
\hline
\end{tabular}

\section{Results}

\section{Patient characteristics}

In this study, a total of 211 subjects were included. Among of them, 89 patients diagnosed with HCC, 44 patients with chronic hepatopathy (21 individuals with chronic hepatitis $\mathrm{B}$, and 23 individuals with $\mathrm{HBV}$-induced liver cirrhosis) were gathered at general surgery department of Anhui Provincial Hospital (Hefei, China) between June 2019 and January 2020. Besides, 78 healthy controls were chosen from the hospital at the same period. The clinical characteristics of these participants recruited were presented in Table 1. For each stage of research, the age and gender of participants were considered fully before adopting.

\section{Screening of candidate miRNAs}

Following the route of the design scheme stated above, the public dataset (GSE104310) was analyzed and the genes of CLEC4G and CLEC4M were found with a significant difference between tumors and controls (Figure S1). To our knowledge, recent studies have shown that this pair of genes is closely associated with the occurrence and progression of cancer, especially in HCC [36,37]. Hence, the study on miRNAs associated with the genes aroused our interests, and we expected to discover some targets more valuable for further research. Finally, 11 candidate miRNAs (Figure S2, Table S3) were obtained using TargetScan Human 7.2 database, as well as Venn diagram tool.

\section{Identification of potential endogenous miRNA references in human serum}

In order to identify potential endogenous controls from the candidate miRNAs, the 11 target miRNAs (Table S3) in serum samples of HCC patients (diagnosed with BCLC stage 0 and $\mathrm{A}, \mathrm{n}=14$ ) and healthy controls $(n=10)$ were tested using RT-qPCR approach, and the distributions of raw $\mathrm{Cq}$ values of these miRNAs was presented in Figure 2. Apparently, the $\mathrm{Cq}$ values of miR-4644 were more concentrated in total samples $(n=24)$, indicating that it might have high stability than other endogenous miRNAs. Meanwhile, multiple indicators, such as standard deviation (SD), interquartile range (IQR), and coefficient of variation $(\mathrm{CV})$ value were also calculated for validating this consequence, and the results of data were shown in Table 2. It was not hard to find that the values of SD, IQR, and CV of miR-4644 were almost the lowest among these endogenous miRNAs; thus, we can conclude that miR-4644 was the most stable one of these candidate endogenous miRNAs. In addition, miR-4644 was also found with considerably lower $\mathrm{Cq}$ value than other miRNAs. In particular, $\mathrm{Cq}$ value varies inversely with gene expression abundance under the same conditions, namely the smaller the $\mathrm{Cq}$ value, the higher the expression abundance [38]. Thus, indicating that miR-4644 was highly expressed in the serum. Without a doubt, high expression abundance was essential for an ideal reference gene. Given the above 
considerations, miR-4644 was proved to be much more stable than other endogenous miRNAs, and it was of great importance for further research if as a valuable endogenous reference.

Table 2. Quantitative results of candidate endogenous miRNAs obtained by qRT-PCR

\begin{tabular}{llll}
\hline miRNA & $\begin{array}{l}\mathrm{Cq}(\text { Total, } \mathrm{n}=24) \\
(\text { Mean } \pm \mathrm{SD})\end{array}$ & $\begin{array}{l}\mathrm{Cq}(\text { Total, } \mathrm{n}=24) \\
\mathrm{IQR}\end{array}$ & $\begin{array}{l}\mathrm{Cq}(\text { Total, } \mathrm{n}=24) \\
(\mathrm{CV}: \% \mathrm{Cq})\end{array}$ \\
\hline miR-3612 & $36.89 \pm 1.50$ & 2.47 & 4.05 \\
miR-1255b-5p & $35.85 \pm 1.98$ & 3.12 & 5.53 \\
miR-1255a & $35.50 \pm 1.26$ & 1.76 & 3.54 \\
miR-4306 & $32.09 \pm 1.05$ & 1.47 & 3.28 \\
miR-4644 & $24.51 \pm 0.45$ & 0.77 & 1.83 \\
miR-185-5p & $34.94 \pm 1.32$ & 1.79 & 3.77 \\
miR-6508-3p & $33.17 \pm 1.80$ & 2.19 & 5.43 \\
miR-650 & $27.53 \pm 1.91$ & 2.93 & 6.92 \\
miR-6510-5p & $28.34 \pm 1.35$ & 1.85 & 4.76 \\
miR-4443 & $26.47 \pm 0.92$ & 1.44 & 3.46 \\
miR-6849-5p & $30.86 \pm 1.53$ & 2.32 & 4.95 \\
\hline
\end{tabular}

\section{Validation of miR-4644 as a normalizer by RT-qPCR}

To validate the stability of $\operatorname{miR}-4644$ as an endogenous normalizer for miRNA quantification in hepatocellular carcinoma, the expression patterns of miR-4644 were further analyzed by RT-qPCR in 42 HCC patients (diagnosed with BCLC stage 0 and A) and 40 healthy controls. In addition, four commonly reference genes, such as miR-16, miR-24, miR-103, and miR-191 reported in serum [22, 39, 40], as well as U6 snRNA provided in the quantitative kit were also used for validating at the same time. Finally, the expression levels of miR-4644, and those genes stated above were shown in Figure 3. There were no significant difference on miR-4644, as well as the other commonly miRNA references between HCC and healthy controls, except for the U6 gene. Besides, we have also found that the average raw $\mathrm{Cq}$ value of miR-4644 was even lower than these commonly reference controls. In other words, the miR-4644 might have a higher abundance of expression than these traditional reference controls. Hence, we deduced that miR-4644 has the potential to be an internal reference control due to its characteristics with excellent stability and expression abundance, and it was necessary for further confirmation.

\section{Stability analysis by software algorithms}

In order to further confirm the stability of miR-4644, and compared its performance with other reference genes, geNorm, NormFinder, and comparative $\Delta \mathrm{Cq}$ programs were adopted for further verification. Finally, the trend chart on the stability of these genes using the geNorm algorithm was presented in Figure 4A, suggesting that miR-4644 was the most stable one among the selected genes. In addition, the stability and ranking of the target genes were shown in Table 3. In particular, the smaller the value of the target gene, the higher the stability, and vice versa [33]. In this program, the expressions of miR-4644 and miR-16 showed higher stability on account of the lower $M$ values, followed were miR-191, miR-103, and miR-24 (Table 3). Generally, the threshold value of $\mathrm{M}$ in geNorm is 1.5, and only when $M$ value less than 1.5 can indicate that the gene with high stability. Here, the $\mathrm{M}$ values of all miRNAs were almost less than 1.5, except for U6. This might mean that it has poor stability on expression in serum. In addition, in terms of determining the optimal number of references, the results of $\mathrm{V}_{2} / \mathrm{V}_{3}, \mathrm{~V}_{3} / \mathrm{V}_{4}$, and $\mathrm{V}_{4} / \mathrm{V}_{5}$ were all less than 0.15 (Figure 4B), suggesting that it was sufficient to select two reference genes for great performance. Hence, both of miR-4644 and miR-16 were the most appropriate choice, and combined them into a whole might better enhance the accuracy of quantitative results. For NormFinder analysis, miR-4644 was also recognized as the most stable reference due to the lowest $M$ value at 0.160 (Figure 5A), and the ranking of other genes was consistent with previous analyses using geNorm (Table 3). Finally, the stability of these genes was deeply verified by the comparative $\Delta \mathrm{Cq}$ procedure. In this analysis, we first calculated the $\Delta \mathrm{Cq}$ values (target gene $\mathrm{Cq}$ - total mean $\mathrm{Cq}$ ) of each sample, then, the standard deviations of the target gene in all selected samples were obtained for evaluating the stability. Here, the trends of stability presented in mean SD were shown in Figure 5B, indicating that miR-4644 remained to be the most stable gene (mean $\mathrm{SD}=0.496$ ) in all reference genes. More importantly, the ranking of stability using this method was the same as those of the previous two analyses (Table 3). Consequently, based on the above findings, we can draw the conclusion that miR-4644 was more stable than the other commonly reference genes, and it can be regarded as a suitable miRNA reference control. Besides, it needs to be stressed that the expression of U6 fluctuated greatly in serum, thus we insisted that it was completely incapable of circulating miRNA analysis owing to this poor stability.

\section{Verification of stability using the public database}

To further validate the stability of expression for miR-4644 and selected miRNA references, a public database, namely BBCancer was analyzed and the similar findings and conclusions stated above were obtained in this database. Figure S3 described the differential expression analyses of blood miRNAs in 13 cancers types. We found that the expression difference of miR-4644 in 13 blood of cancers types 
was significantly lower other commonly miRNA references when relative to the normal tissue. Apparently, the same results could be presented more visually in heat map (Figure S4). Therefore, it can be concluded that the stability of miR-4644 was higher than that of the commonly miRNA references. Furthermore, miR-4644 was probably an ideal housekeeping gene by reason of its higher stability of expression between tissue and blood.

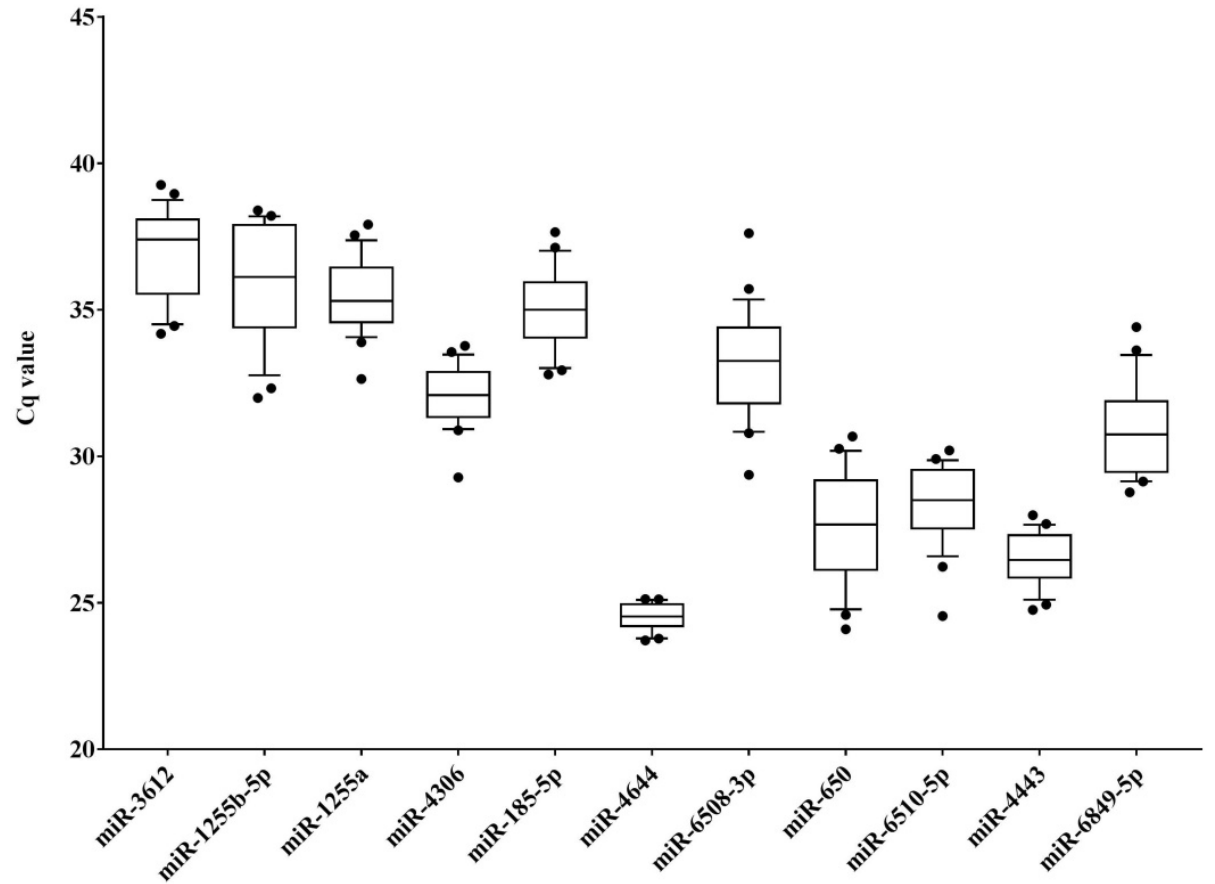

Figure 2. Distribution of raw cycle $(\mathrm{Cq})$ values of candidate miRNAs in serum samples (HCC: $n=14$; healthy: $n=10$ ). Data are shown as box and whiskers plot (10th -90 th percentile), the dots represented outliers of $C_{q}$ values.
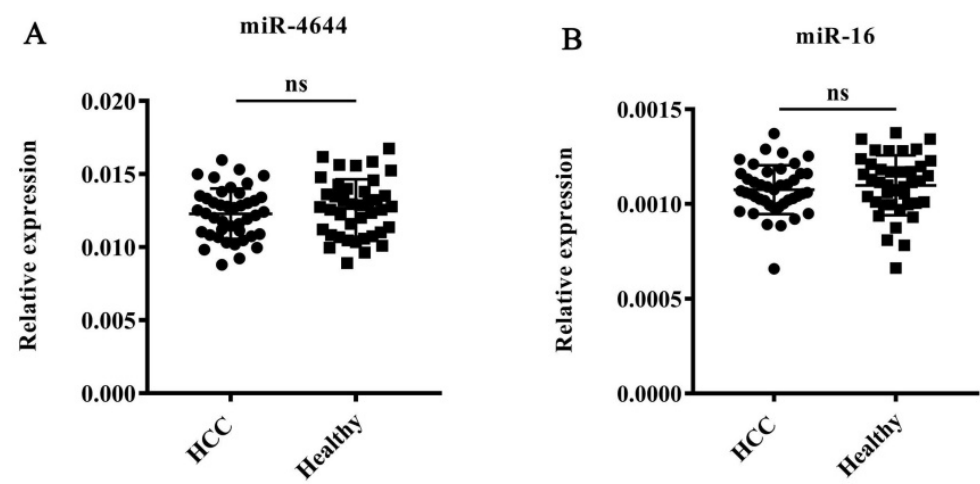

C $\quad \operatorname{miR-24}$
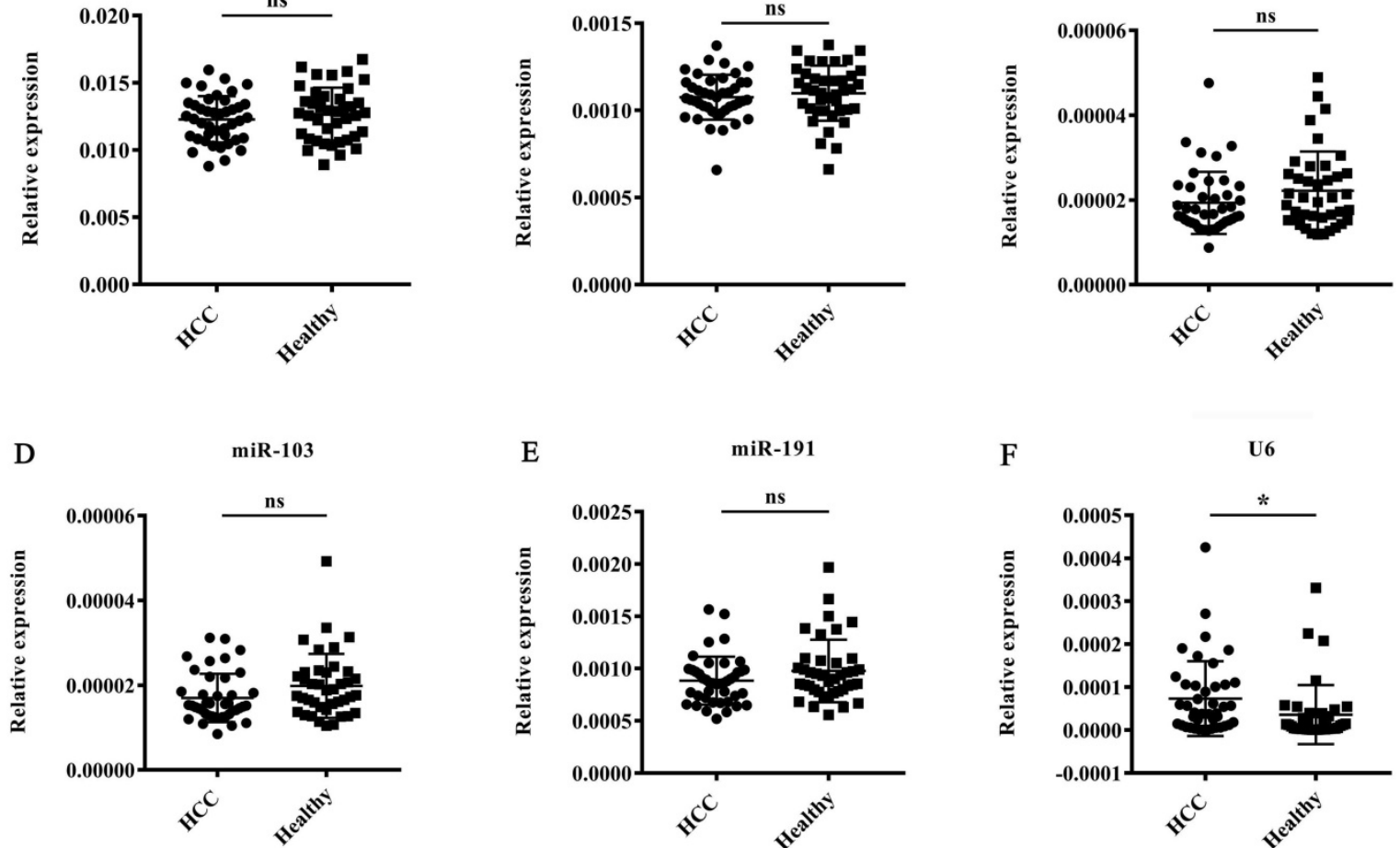

Figure 3. Expression patterns of miR-4644, and other five known reference genes in HCC and health groups (HCC: $n=42 ;$ healthy: $n=40)$. The expression levels were normalized to spiked cel-miR-67. (A) miR-4644, (B) miR-16, (C) miR-24, (D) miR-103, (E) miR-191, (F) U6. Data are shown as means \pm SD, ns = not significant, $* p<0.05$. 


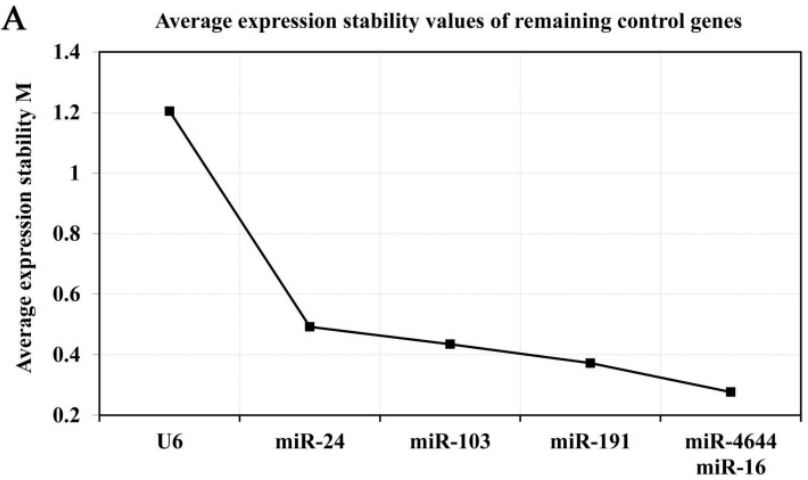

$<::::$ Least stable genes Most stable genes ::::>

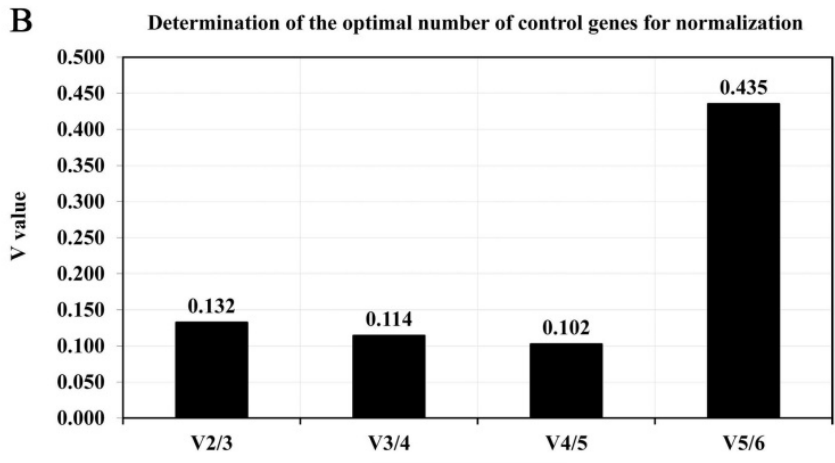

Pairwise Variations

Figure 4. Expression stability and the optional number of reference genes by geNorm analysis. (A) Average values of expression stability for remaining controls. (B) Pairwise variation coefficient ( $V$ value) for determining the number of optimal references.
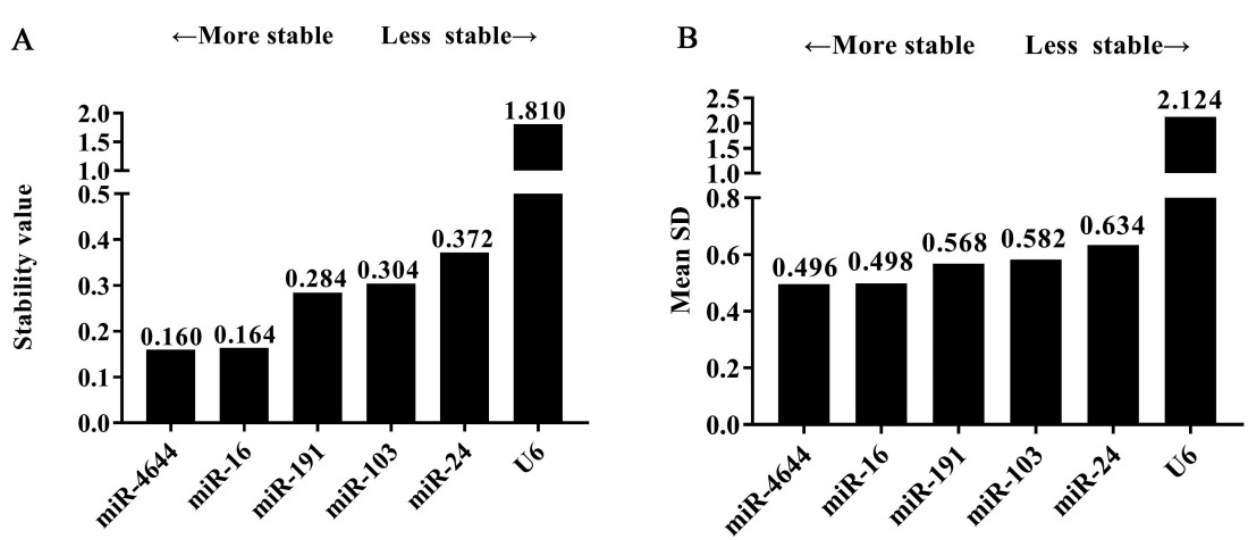

Figure 5. The tendency of gene stability analyzed using software algorithms. (A) NormFinder program. (B) Comparative $\Delta$ Cq program.

Table 3. The overall ranking according to the calculation of stability values using different analytical tools

\begin{tabular}{llllllll}
\hline $\begin{array}{l}\text { Overall } \\
\text { Ranking }\end{array}$ & Gene & \multicolumn{2}{c}{ geNorm } & \multicolumn{2}{c}{ NormFinder } & \multicolumn{2}{c}{$\begin{array}{c}\text { Comparative } \\
\Delta \text { Cq }\end{array}$} \\
\cline { 3 - 8 } & & \multicolumn{3}{c}{$\begin{array}{c}\text { M } \\
\text { value }\end{array}$} & $\begin{array}{c}\text { Ranking } \\
\text { Stability } \\
\text { value }\end{array}$ & $\begin{array}{c}\text { Ranking } \\
\text { Mean } \\
\text { SD }\end{array}$ & Ranking \\
\hline 1 & miR-4644 & 0.857 & 1 & 0.160 & 1 & 0.496 & 1 \\
2 & miR-16 & 0.858 & 2 & 0.164 & 2 & 0.498 & 2 \\
5 & miR-24 & 0.977 & 5 & 0.372 & 5 & 0.634 & 5 \\
4 & miR-103 & 0.968 & 4 & 0.304 & 4 & 0.582 & 4 \\
3 & miR-191 & 0.946 & 3 & 0.284 & 3 & 0.568 & 3 \\
6 & U6 & 2.710 & 6 & 1.810 & 6 & 2.124 & 6 \\
\hline
\end{tabular}

\section{Application of miRNA combinations in expanded types of chronic hepatopathy}

In order to confirm the usability of miRNA combination as a reference control for quantification of circulating miRNAs, we combined miR-4644 and miR-16 into a whole for further validation. In this stage, 105 additional donors, including 33 patients with diagnosed HCC (confirmed with BCLC stage B or C), 21 patients with $\mathrm{CHB}, 23$ patients with $\mathrm{HBV}$ induced liver cirrhosis, and 28 healthy controls were involved into the test. The expression levels of miR4644, miR-16, and their combination across the above four types were illustrated in Figure 6. In particular, for comparing the performance differences among them when as the reference control, the relative expression level of each one was normalized to the other two references. Finally, no significant difference $(p>0.05)$ among four groups was found in miR-4644, miR-16, and their combination, when normalized to any two of them. Thus, suggesting that both of miR4644 and miR-16 have high stability of expression in expanded types of chronic hepatopathy. Furthermore, the relative expression difference of miR-4644, miR-16, and their combination could be distinguished from $p$ value calculated by one-way ANOVA and Nonparametric tests. On the one hand, the $P$ value of individuals (such as miR-4644, shown in Figure 6B) normalized to the combination was greater than it normalized to the miR-16 (Figure 6A) among the four subjects. Similarly, the same was true for the miR-16 (Figure 6D, 6C). Thus, it can be suggested that the performance of the combination was better than any one of the individuals if treated as the reference control. In other words, the accuracy of quantitative results would be improved if combined reference individuals into a whole. On the other hand, the $p$ value of combination normalized to the miR4644 (Figure 6F) was greater than it normalized to the 
miR-16 (Figure 6E); this proved again that the miR4644 was superior to miR-16 when as the reference control. In summary, the combination consisted of miR-4644 and miR-16 was demonstrated to be a suitable reference control, and it would contribute to the achievement of better effect for miRNAs analyses.

Frankly speaking, while we have seen the remarkable performance of miRNA combination as a suitable reference control, combined which individuals into a whole could receive better results were not clear. Hence, in order to explore this doubt, several miRNA references used above were made into combinations; the stability of these combinations was also evaluated by geNorm, NormFinder, and comparative $\Delta \mathrm{Cq}$ tools. And the comparisons of stability in four combinations selected were shown in Figure 7. Firstly, the same results were achieved for the ranking of four combinations when analyzed using different programs. Furthermore, the values of the combination of has-miR-4644 and has-miR-16 were all less than other combinations, indicating that the combination of has-miR-4644 and has-miR-16 that has similar stability was greater than that with a greater difference in stability. More importantly, we found that the values of miRNA combinations (miR-4644 \& 191, miR-4644 \& 103, miR-4644 \& 24) with a greater difference have little difference in the stability (Figure 7A-C). However, the value of the combination (miR-4644 \& 16) was significantly less than other three combinations (Figure 7B, 7C). Therefore, it was not hard to see that the combination of miRNA references with comparable stability might be more suitable for a reference control, and only in this way can greatly enhance the accuracy of miRNA quantification.

\section{Discussion}

Currently, the liquid biopsy-based on miRNA biomarkers have been written in the Guidelines for Diagnosis and Treatment of Primary Liver Cancer in
A $\quad \begin{gathered}\text { miR-4644 } \\ \text { normalized to miR-16 }\end{gathered}$

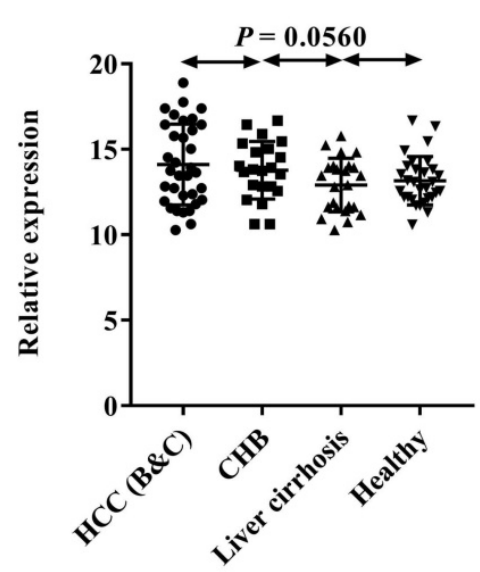

D miR-16
normalized to miRNA combination

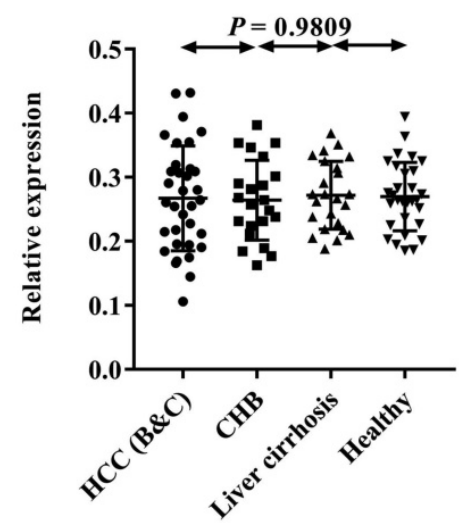

B

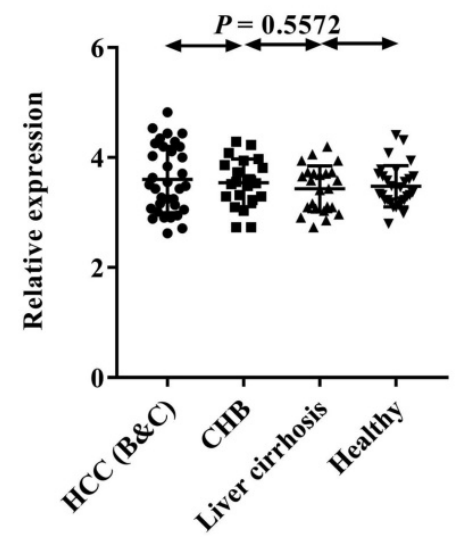

E

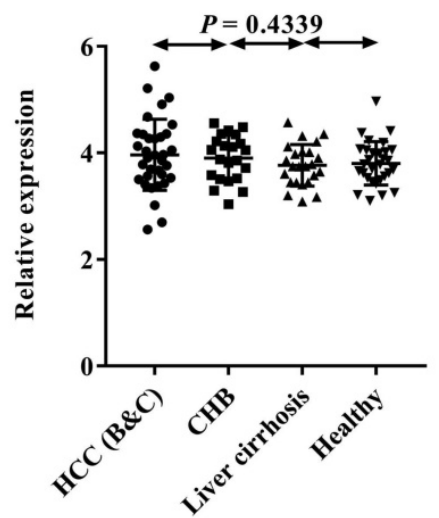

C

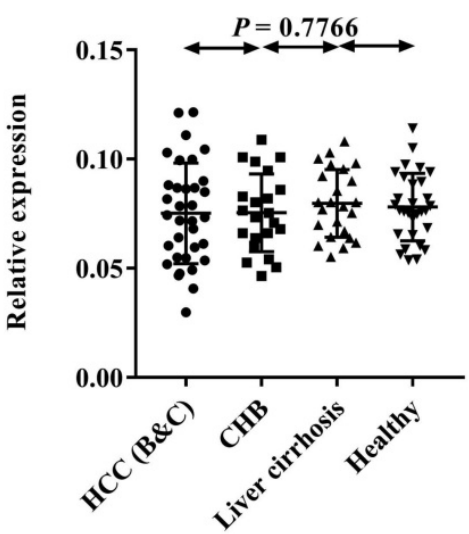

F

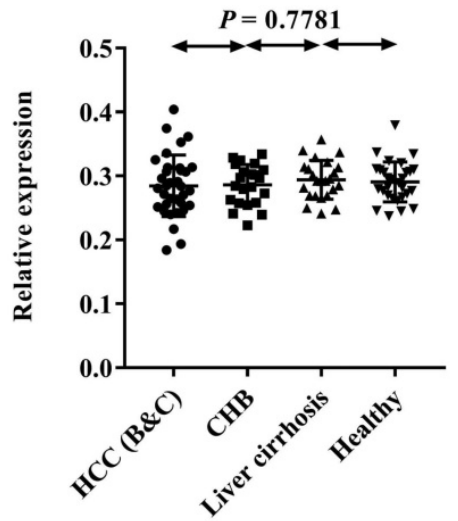

Figure 6. Expression levels of endogenous miRNAs and their combinations by RT-qPCR. The relative expression levels of each gene was normalized to other two references between HCC patients (stage B or $C, n=33)$, CHB $(n=21)$, liver cirrhosis $(n=23)$, and healthy controls $(n=28)$. $(A)$ miR-4644 normalized to miR-16. (B) miR-4644 normalized to their combination. (C) miR-16 normalized to miR-4644. (D) miR-16 normalized to miRNA combination. (E) miRNA combination normalized to miR-16. (F) miRNA combination normalized to miR-4644. Data are shown as means \pm SD. 
China (2019 edition), and the experts believed that the diagnostic protocol would play a vital part in early diagnosis and efficacy evaluation for HCC. Therefore, it is of profound significance for achieving accurate quantification of miRNAs for clinical applications in the future. However, there is still no general consensus until now on reliable endogenous references for normalization of miRNAs quantification [39]. Particularly for endogenous miRNA control in HCC, there was a dispute among researchers. For instance, $\mathrm{Hu}$ et al. discovered and considered miR-1228 as a stable endogenous control for the quantification of plasma miRNAs in patients with hepatopathy [27]. However, this so-called invariant miR-1228 was undetectable in an array assay of Lin et al. [28], and they had to use synthetic cel-miR-67 to analyze serum miRNA levels. To our knowledge, it was probably due to the different types of samples they tested. Without a doubt, there were few reports until now on the identification of miRNA reference in the serum of HCC, thus will seriously affect the accuracy of miRNA quantification in serum. Hence, it is particularly urgent to search for an optimal endogenous miRNA normalizer in the serum of HCC.

Hence, we aimed to identify a satisfactory endogenous miRNA reference for the quantification of circulating miRNAs in the serum of HCC. Frankly speaking, in this study, the new discovery of miR-4644 as a miRNA normalizer might be a certain amount of chance and luck. Initially, a pair of key genes, namely CLEC4G and CLEC4M were found with significant difference between tumors and controls, when retrieved a public dataset (GSE104310) of hepatocellular carcinoma. And to our knowledge, this pair of genes has been proved to be closely associated with the occurrence and progression of cancer, even in the HCC. Besides, the majority of genes were regulated by the miRNAs. Thus, the miRNAs associated with the genes aroused our interests, and we expected to discover some miRNAs valuable for further research. Finally, miR-4644 stood out from the candidate miRNAs, and was deemed as an ideal normalizer due to its less variability and high abundance in expression. Thus, it was well worthy of further study.

Afterwards, in order to evaluate and confirm the reliability of miR-4644 for a reference normalizer, two measures were adopted for this validation phase. On one hand, a total of 82 samples (HCC patients, $n=42$; healthy controls, $n=40$ ) were included in the assay for reducing the influence of certain chance factors on the effects of experiment. On the other hand, several commonly reference controls reported in the serum of other diseases were selected and tested in this study together $[23,41]$. We aim to compare the difference between miR-4644 and those known references in stability. Thus, the reliability and stability of miR-4644 as a reference normalizer were guaranteed. Finally, our results indicated that no significant difference was observed in the expression of these genes between $\mathrm{HCC}$ and health groups. Importantly, the raw $\mathrm{Cq}$ values of miR-4644 were also found to be lower than the conventional reference controls in the same circumstances, and generally the lower $\mathrm{Cq}$ values associated with the higher abundance [38]. Hence, we can conclude that the expression abundance of miR-4644 was higher than other commonly reference controls. Without a doubt, the high expression abundance was important for an ideal reference gene [42]. Presently, to our knowledge, miR-16 was one of the known reference controls with the highest expression abundance $[43,44]$, but at the moment, its advantage had lost in this respect, and might be replaced by miR-4644. To our knowledge, this was the first report that a new endogenous miRNA (miR4644) has higher expression abundance than any other miRNAs.

Certainly, the stability of miR-4644, as well as other known references was evaluated using
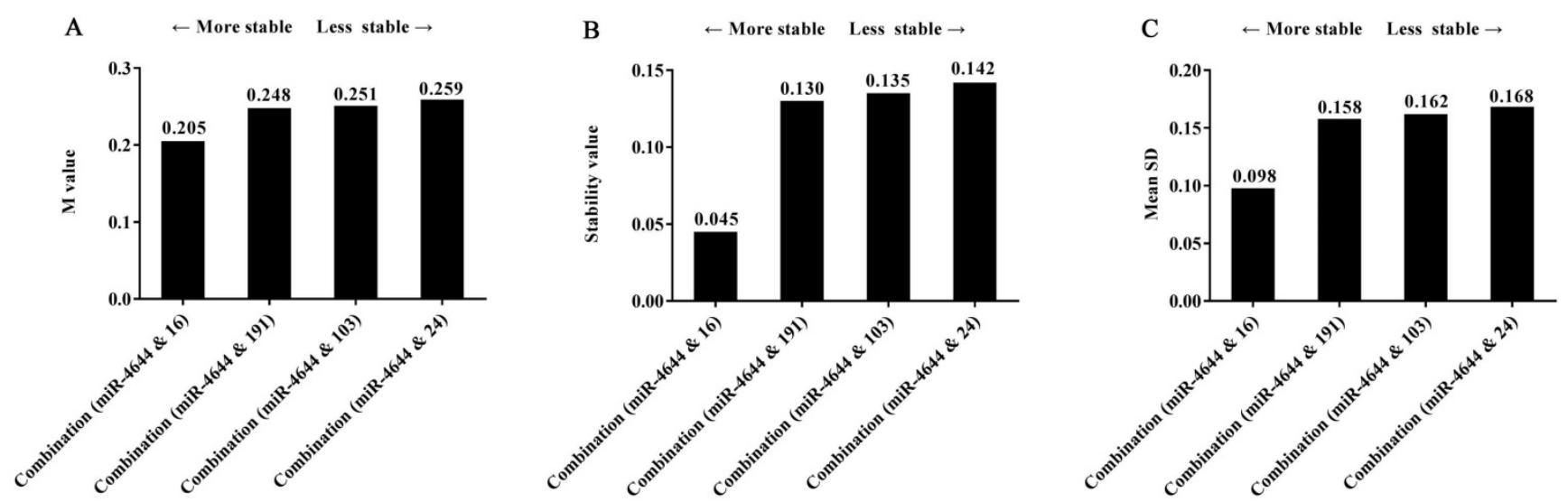

Figure 7. The stability of four combinations analyzed using different algorithms. (A) geNorm program. (B) NormFinder program. (C) Comparative $\Delta$ Cq program. 
geNorm [33], NormFinder [34], and comparative $\Delta \mathrm{Cq}$ procedures [35]. Thus, the performance of these reference genes could be confirmed in different algorithms. Unlike the previous studies on the identification of other miRNA normalizers, the ranking of miR-4644 was fairly stable, indicating that the reliability of the results was verified. Interestingly, geNorm tool suggested that optimal performance could be reached if combined two genes into a whole. To our knowledge, there was a precedent on the research of miRNA combination as promising biomarker, and it has been demonstrated that this combination possessed high diagnostic accuracy in HCC diagnosis [45]. But that was not known yet for the combination of miRNA references. Hence, the study on performance of combination of miRNA references aroused our interests. In this study, miR-4644 and miR-16 were considered to be ranked in the top two in stable genes. So, this aroused our great interests in exploring this issue. In order to evaluate the stability on a combination of miRNAs (miR-4644 and miR-16), and expand its application in other types of chronic hepatopathy, another set of additional subjects, including patients diagnosed with stage $\mathrm{B}$ or $\mathrm{C}$ of $\mathrm{HCC}$, with $\mathrm{CHB}$, with liver cirrhosis, as well as healthy controls were chosen in this testing. In this phase of research, to compare the difference of performance between two references individuals and their combination as much as possible, we broke the conventional thinking mode, and firstly proposed a cross-normalization approach for analysis of reference genes. Namely, the relative expression level of each gene was normalized to any one of the other references in the group. Thus, the difference among them could be easily obtained through the $p$ value using one-way ANOVA and nonparametric tests. Generally, the larger the $p$ value, the less significant the difference, namely the stability was better. Without a doubt, the threshold value of $p$ was 0.05 , and the scheme was valid only if the value is greater than 0.05 for this project. In particular, the strategy might only apply to study on the difference of expression level for reference genes. Finally, the results were clear that the combination was demonstrated with great performance than miRNA individuals. Therefore, we can conclude that the combination of miR-4644 and miR-16 would be an ideal reference normalizer for circulating miRNA quantification. It should be noted that it was indeed a novel strategy for quantitative standardization when combined multiple references into a whole, thus can really improve the accuracy of quantification [46]. However, a new question arose, namely which individuals were combined into a whole would give rise to amplification effect in their combinations. In order to explore the selectivity of miRNA references in the combinations, several miRNA individuals with different stability were combined separately for further study. The stability of these combinations was also validated using geNorm, NormFinder, and comparative $\Delta \mathrm{Cq}$ procedures, and it was not hard to see that the combination of miR-4644 and miR-16 was much more stable than other combinations. In briefly, these two miRNA references with closer stability could really give rise to an amplification effect in the respect of stability. In our opinion, the results suggested that an ideal reference combination should meet two essential demands: (1) the combination must consist of two reference genes which ranking the top in stability, (2) the stability is broadly similar across the individuals. Certainly, we guessed this conclusion might apply equally to reference controls of the other genes.

We acknowledge that there were still some limitations in this study. In terms of endogenous miRNAs normalizers in HCC, although our study has proved the miR-4644 was an ideal control in serum samples, its expression level and stability in the plasma sample were unknown. We emphasized this point because of miR-1280 as a reference control in plasma reported by $\mathrm{Hu}$ et al., but this was undetectable in an array of serum [27, 28], nevertheless, a new study has suggested that miR-1280 could be used as a serum normalizer in HCC [47]. Furthermore, we have paid attention to the average $\mathrm{Cq}$ value of miR-1280 was greater than miR-4644 we identified. As the principle of the higher the value, the lower the abundance, hence, we can conclude that miR-4644 has higher abundance than miR-1280, and it might be more suitable for the normalizer in HCC.

\section{Conclusions}

In this study, miR-4644 was demonstrated as a suitable endogenous miRNA reference with less expression variability and higher expression abundance in serum. Thus, it will contribute to accurate quantification of miRNA in HCC research. Meanwhile, further research has suggested that the reference combination of miR-4644 and miR-16 has fairly higher stability than each of them, and we also believed that a combination consisted of two reference individuals with similar characteristics in stability would give rise to amplification effect. In the long run, an increasing number of new miRNA normalizers will be identified, then the accuracy of miRNA quantification could be greatly improved if follow this principle. 


\section{Supplementary Material}

Supplementary figures and tables. http://www.jcancer.org/v11p7032s1.pdf

\section{Acknowledgements}

We would like to thanks prof. Wei-Dong Jia (Anhui Provincial Hospital) for providing blood samples of subjects. We sincerely appreciate the RNA sequencing data provided by Jing-Ping Yun and his team. This study was developed at the Anhui Institute of Optics and Fine Mechanics, Hefei Institutes of Physical Science, Chinese Academy of Sciences, and supported by the Science and Technology Major Program of Anhui, China (No.18030801139), the Science and Technology Service Network Initiative of Chinese Academy of Sciences (No.KFJ-STS-ZDTP063).

\section{Competing Interests}

The authors have declared that no competing interest exists.

\section{References}

1. Bray F, Ferlay J, Soerjomataram I, et al. Global cancer statistics 2018: GLOBOCAN estimates of incidence and mortality worldwide for 36 cancers in 185 countries. CA Cancer J Clin. 2018; 68: 394-424.

2. Chen W, Zheng R, Baade PD, et al. Cancer statistics in China, 2015. CA Cancer J Clin. 2016; 66: 115-132.

3. Cheng S, Chen M, Cai J, et al. Chinese Expert Consensus on Multidisciplinary Diagnosis and Treatment of Hepatocellular Carcinoma with Portal Vein Tumor Thrombus (2018 Edition). Liver Cancer. 2020; 9: 28-40.

4. Segal R, Miller K, Jemal A. Cancer statistics, 2018. CA Cancer J Clin. 2018; 68: 7-30.

5. Bruix J, Sherman M, Committee PG. American Association for the Study of Liver DiseasesManagement of hepatocellular carcinoma. An Update. 2011; 53: 1020-1022.

6. Luo $\mathrm{P}, \mathrm{Wu}$ S, Yu Y, et al. Current status and perspective biomarkers in AFP negative HCC: towards screening for and diagnosing hepatocellular carcinoma at an earlier stage. Pathol Oncol Res. 2020; 26: 599-603.

7. Abrams T, Ben-Josef E, Bloomston $\mathrm{P}$, et al. NCCN clinical practice guidelines in oncology: hepatobiliary cancers. J Natl Compr Canc Netw. 2009; 7: 350-391.

8. Huang J, Chen W, Yao S. Assessing diagnostic value of contrast-enhanced ultrasound and contrast-enhanced computed tomography in detecting small hepatocellular carcinoma: A meta-analysis. Medicine. 2017; 96: e7555.

9. Di Tommaso L, Spadaccini M, Donadon M, et al. Role of liver biopsy in hepatocellular carcinoma. World J Gastroenterol. 2019; 25: 6041-6052.

10. Berrien-Elliott MM, Sun Y, Neal C, et al. MicroRNA-142 is critical for the homeostasis and function of type 1 innate lymphoid cells. Immunity. 2019; 51: 479-490. e6.

11. Xie $\mathrm{X}, \mathrm{Hu} \mathrm{H}$, Tong $\mathrm{X}$, et al. The mTOR-S6K pathway links growth signalling to DNA damage response by targeting RNF168. Nat Cell Biol. 2018; 20: 320-331.

12. Nikpay M, Beehler K, Valsesia A, et al. Genome-wide identification of circulating-miRNA expression quantitative trait loci reveals the role of several miRNAs in the regulation of cardiometabolic phenotypes. Cardiovasc Res. 2019; 115: 1629-1645.

13. Szabo G, Bala S. MicroRNAs in liver disease. Nat Rev Gastro Hepat. 2013; 10: 542-552

14. Cao D, Di M, Liang J, et al. MicroRNA-183 in Cancer Progression. J Cancer. 2020; 11: 1315-1324.

15. Lawrie $\mathrm{CH}, \mathrm{Gal}$ S, Dunlop HM, et al. Detection of elevated levels of tumour-associated microRNAs in serum of patients with diffuse large B-cell lymphoma. Br J Haematol. 2008; 141: 672-675.
16. Min L, Zhu S, Chen L, et al. Evaluation of circulating small extracellular vesicles derived miRNAs as biomarkers of early colon cancer: a comparison with plasma total miRNAs. J Extracellr Vesicles. 2019; 8: 1643670.

17. Asano N, Matsuzaki J, Ichikawa M, et al. A serum microRNA classifier for the diagnosis of sarcomas of various histological subtypes. Nat Commun. 2019; 10: 1-10.

18. Keller A, Fehlmann T, Backes $\mathrm{C}$, et al. Competitive learning suggests circulating miRNA profiles for cancers decades prior to diagnosis. RNA Biol. 2020; 16: 1-11.

19. Shigeyasu K, Toden S, Zumwalt TJ, et al. Emerging role of microRNAs as liquid biopsy biomarkers in gastrointestinal cancers. Clin Cancer Res. 2017; 23: 2391-2399.

20. Hellemans J, Vandesompele J. Selection of reliable reference genes for RT-qPCR analysis. In: Quantitative Real-Time PCR, Methods in Molecular Biology. Switzerland: Springer Nature. 2014; p:19-26.

21. Xiang M, Zeng Y, Yang R, et al. U6 is not a suitable endogenous control for the quantification of circulating microRNAs. Biochem Biophys Res Commun. 2014; 454: 210-214.

22. Donati S, Ciuffi S, Brandi ML. Human circulating miRNAs real-time qRT-PCR-based analysis: an overview of endogenous reference genes used for data normalization. Int J Mol Sci. 2019; 20: 4353.

23. Madadi S, Schwarzenbach H, Lorenzen J, et al. MicroRNA expression studies: challenge of selecting reliable reference controls for data normalization. Cell Mol Life Sci. 2019; 76: 3497-3514.

24. Huang Z, Chen W, Du Y, et al. Serum miR-16 as a potential biomarker for human cancer diagnosis: results from a large-scale population. J Cancer Res Clin Oncol. 2019; 145: 787-796.

25. Pagacz K, Kucharski P, Smyczynska U, et al. A systemic approach to screening high-throughput RT-qPCR data for a suitable set of reference circulating miRNAs. BMC Genomics. 2020; 21: 111

26. Kroh EM, Parkin RK, Mitchell PS, et al. Analysis of circulating microRNA biomarkers in plasma and serum using quantitative reverse transcription-PCR (qRT-PCR). Methods. 2010; 50: 298-301.

27. Hu J, Wang Z, Liao BY, et al. Human miR-1228 as a stable endogenous control for the quantification of circulating microRNAs in cancer patients. Int J Cancer. 2014; 135: 1187-1194.

28. Lin X-J, Chong Y, Guo Z-W, et al. A serum microRNA classifier for early detection of hepatocellular carcinoma: a multicentre, retrospective, longitudinal biomarker identification study with a nested case-control study. Lancet Oncol. 2015; 16: 804-815.

29. Drees EE, Pegtel DM. Circulating miRNAs as Biomarkers in Aggressive B Cell Lymphomas. Trends in cancer. 2020; 06: 1-14.

30. Agarwal V, Bell GW, Nam J-W, et al. Predicting effective microRNA target sites in mammalian mRNAs. elife. 2015; 4: e05005.

31. Zuo Z, Hu H, Xu Q, et al. BBCancer: an expression atlas of blood-based biomarkers in the early diagnosis of cancers. Nucleic Acids Res. 2020; 48: 789-796.

32. Ferracin M, Negrini M. Quantification of circulating microRNAs by droplet digital PCR. In: Digital PCR, Methods in Molecular Biology. Switzerland Springer Nature. 2018; p: 445-457.

33. Vandesompele J, De Preter K, Pattyn F, et al. Accurate normalization of real-time quantitative RT-PCR data by geometric averaging of multiple internal control genes. Genome Biol. 2002; 3: research0034. 1.

34. Andersen C, Ledet-Jensen J, Orntoft T. Normalization of real-time quantitative RT-PCR data: a mode-based variance estimation approach to identify genes suited for normalization, applied to bladder and colon cancer data sets. Cancer Res. 2004; 64: 5245-5250.

35. Schmittgen TD, Livak KJ. Analyzing real-time PCR data by the comparative C T method. Nat Protoc. 2008; 3: 1101-1108.

36. Sarathi A, Palaniappan A. Novel significant stage-specific differentially expressed genes in hepatocellular carcinoma. BMC Cancer. 2019; 19: 663.

37. Melis M, Diaz G, Kleiner DE, et al. Viral expression and molecular profiling in liver tissue versus microdissected hepatocytes in hepatitis B virus-associated hepatocellular carcinoma. J Transl Med. 2014; 12: 230.

38. Li M, Xie F, He Q, et al. Expression Analysis of XTH in Stem Swelling of Stem Mustard and Selection of Reference Genes. Genes. 2020; 11: 113.

39. Schwarzenbach H, Da Silva AM, Calin G, et al. Data normalization strategies for microRNA quantification. Clin Chem. 2015; 61: 1333-1342.

40. Gharbi S, Shamsara M, Khateri S, et al. Identification of reliable reference genes for quantification of microRNAs in serum samples of sulfur mustard-exposed veterans. Cell J. 2015; 17: 494-501.

41. Hunsaker M, Barba G, Kingsley K, et al. Differential microRNA expression of miR-21 and miR-155 within oral cancer extracellular vesicles in response to melatonin. Dent J. 2019; 7: 48.

42. Connors E, Soto-Dávila M, Hossain A, et al. Identification and validation of reliable Aeromonas salmonicida subspecies salmonicida reference genes for differential gene expression analyses. Infect Genet Evol. 2019; 73: 314-321 
43. Hartung A, Makarewicz O, Egerer R, et al. EBV miRNA expression profiles in different infection stages: A prospective cohort study. PLoS One. 2019; 14: e0212027.

44. Pritchard CC, Kroh E, Wood B, et al. Blood cell origin of circulating microRNAs: a cautionary note for cancer biomarker studies. Cancer Prev Res. 2012; 5: 492-497.

45. Ji J, Chen H, Liu X, et al. A miRNA Combination as Promising Biomarker for Hepatocellular Carcinoma Diagnosis: A Study Based on Bioinformatics Analysis. J Cancer. 2018; 9: 3435-3446.

46. Nilsen A, Jonsson M, Aarnes E-K, et al. Reference MicroRNAs for RT-qPCR Assays in Cervical Cancer Patients and Their Application to Studies of HPV16 and Hypoxia Biomarkers. Transl Oncol. 2019; 12: 576-584.

47. Yogi PM, Luisa C, Claudio T, et al. Selection and validation of miR-1280 as a suitable endogenous normalizer for qRT-PCR Analysis of serum microRNA expression in Hepatocellular Carcinoma. Sci Rep. 2020; 10: 3128 